\title{
THE LAGRANGIAN GAUSS IMAGE OF A SURFACE IN EUCLIDEAN 3-SPACE
}

\author{
MAREK KOSSOWSKI
}

\begin{abstract}
We describe a correspondence between special nonimmersed surfaces in Euclidean 3-space and exact Lagrangian immersions in the cotangent bundle of the unit sphere. This provides several invariants for such nonimmersive maps: the degree of the Gauss map, the Gauss-Maslov class, and the polarization index. The objectives of this paper are to compare these invariants in the cases where the underlying map immerses or fails to immerse and to describe the extend to which these invariants can be prescribed.
\end{abstract}

\section{INTRODUCTION}

Given an immersion $i: M \rightarrow \mathbf{E}^{3}$ of a compact orientable surface into Euclidean 3-space, there is a unique associated exact Lagrangian immersion into the cotangent bundle of the 2-sphere, $\tilde{i}: M \rightarrow T^{*} S^{2 *} \stackrel{\rho}{\rightarrow} S^{2 *}$. The Gauss map $G: M \rightarrow S^{2 *}$ of the immersion can then be recovered from the composite $G=\rho \circ \tilde{i}$. Conversely, given an exact Lagrangian immersion $\tilde{j}: L \rightarrow T^{*} S^{2 *}$ of a compact orientable surface into the cotangent bundle of the sphere, there exists a smooth but not necessarily immersive map $j: L \rightarrow \mathbf{E}^{3}$ with Gauss map defined by $\rho \circ \tilde{j}: L \rightarrow S^{2 *}$. Thus, we have a correspondence between smooth maps of surfaces in Euclidean space which may fail to be immersive yet admit a smooth global Gauss map, and smooth exact Lagrangian immersions into the cotangent bundle of the sphere. It follows that invariants for the Lagrangian Gauss image in $T^{*} S^{2 *}$ also belong to the underlying map into $\mathbf{E}^{3}$. This yields the following global invariants for such maps: the $G$-degree, the Gauss-Maslov class, and the polarization index. The objectives of this paper are to compare these invariants in the cases where the underlying map into $\mathbf{E}^{3}$ immerses or fails to immerse (Theorems 2-4) and to describe the extent to which these invariants can be prescribed. In particular, Theorem 4 indicates that any integer and integral cohomology class can be realized as the $G$-degree and Gauss-Maslov class of a Lagrangian Gauss image. As an application we find new global restrictions on the critical values of the Gauss map for an immersion in $\mathbf{E}^{3}$ (see [K2]). We also find obstructions to global solutions of spherical Monge-Ampère PDE (see [K3]). For example, if $j: L \rightarrow \mathbf{E}^{3}$ has constant mean curvature at every immersive point then the Lagrangian Gauss image $\tilde{j}: L \rightarrow T^{*} S^{2 *}$ has trivial

Received by the editors June 5, 1990 and, in revised form, October 11, 1990 and November 15, 1990.

1980 Mathematics Subject Classification (1985 Revision). Primary 53C20, 53C22, 53C42, 57C45.

Research partially supported by NSF Grant DMS 88-03585. 
Gauss-Maslov class. Similarly, if $j: L \rightarrow \mathbf{E}^{3}$ has constant negative Gauss curvature at every immersive point then the polarization index of $\tilde{j}$ is carried by a cohomology class.

The paper begins with an informal description of the correspondence between nonimmersive maps into $\mathbf{E}^{3}$ and their Lagrangian Gauss image in $T^{*} S^{2 *}$. This is followed by an informal definition of the contact collar over an embedding $i: M \rightarrow \mathbf{E}^{3}$. This construction is used to convert exact Lagrangian immersions in $T^{*} M$ into exact Lagrangian immersions in $T^{*} S^{2 *}$. This in turn provides us with explicit geometric examples of nonimmersive maps into $\mathbf{E}^{3}$ whose invariants are readily computed. These informal definitions will enable the reader to understand the content of Theorems $1-4$. The reader who wishes to study the proofs of Theorems 1-4 will find a detailed discussion of this correspondence and related constructions in the Appendix. The author would like to thank M. Adams, R. L. Bryant, R. B. Gardner, R. Harvey, C. McCrory, T. Shifrin, and R. Varley for inspiration.

\section{Preliminaries}

We let $\langle$,$\rangle denote the inner product on \mathbf{E}^{3}$. Let $\pi: T^{*} \mathbf{E}^{3} \rightarrow \mathbf{E}^{3}$ denote the cotangent bundle of $\mathbf{E}^{3}$ with canonical projection $\pi$ and canonical 1-form $\pi \theta^{c}$. Since $\mathbf{E}^{3}$ carries a flat connection, we have a horizontal projection $\rho: T^{*} \mathbf{E}^{3} \rightarrow$ $\mathbf{E}^{3 *}$, which is defined by parallel translation. Now via double dualization we have an associated horizontal canonical 1-form $\rho \theta^{c}$. Let $S^{2 *} \subset \mathbf{E}^{3 *}$ denote the unit sphere. The set $\rho^{-1}\left(S^{2 *}\right)$ is a product $\mathbf{E}^{3} \times S^{2 *}$. In the Appendix we show that $\rho^{-1}\left(S^{2 *}\right)$ is a contact manifold with respect to the 1 -form $\pi \theta^{c}$, which admits a symplectic transverse section $h: T^{*} S^{2 *} \rightarrow \rho^{-1}\left(S^{2 *}\right) \subset T^{*} \mathbf{E}^{3}$. The map $\rho \circ h: T^{*} S^{2 *} \rightarrow S^{2 *} \subset \mathbf{E}^{3 *}$ agrees with the canonical projection $T^{*} S^{2 *} \rightarrow S^{2 *}$, which we also denote by $\rho$. Throughout this paper, $i: M \rightarrow \mathbf{E}^{3}$ will denote a smooth immersion and $j: L \rightarrow \mathbf{E}^{3}$ will denote a smooth but not necessarily immersive map.

Given a smooth immersion $i: M \rightarrow \mathbf{E}^{3}$ with unit normal $\eta$, we get a map $\tilde{\eta}: M \rightarrow \rho^{-1}\left(S^{2 *}\right) \subset T^{*} \mathbf{E}^{3}$ defined by $\tilde{\eta}(m)=\langle\eta(m),-\rangle \in T_{i(m)}^{*} \mathbf{E}^{3}$. Projection of $\tilde{\eta}$ into the transverse section $h\left(T^{*} S^{2 *}\right)$ defines the Lagrangian Gauss image $\tilde{i}: M \rightarrow T^{*} S^{2 *}$. Now if we are given a $\rho \theta^{c}$-exact Lagrangian immersion $\tilde{j}: L \rightarrow$ $T^{*} S^{2 *}$ then there exists a lift to the contact manifold $\rho^{-1}\left(S^{2 *}\right)$ which pulls the 1-form $\pi \theta^{c}$ back to zero. The $\pi$-projection of this lifted map defines an associated map $j: L \rightarrow \mathbf{E}^{3}$, whose $S^{2 *}$-valued Gauss map is given by $\rho \circ \tilde{j}$. (For details see the Appendix.)

The following is a convenient way to construct Lagrangian immersions into $T^{*} S^{2 *}$. Given an embedding $i: M \rightarrow \mathbf{E}^{3}$ there is an associated contact collar $i_{\mathrm{cc}}: T^{*} M \times(-\varepsilon, \varepsilon) \rightarrow T^{*} \mathbf{E}^{3}$. This is an embedded contact manifold with respect to the 1-form $\pi \theta^{c}$. Now, given a smooth exact Lagrangian immersion $k: L \rightarrow$ $T^{*} M$, there exists a lift to $i_{\mathrm{cc}}\left(T^{*} M \times(-\varepsilon, \varepsilon)\right) \subset T^{*} \mathbf{E}^{3}$ which pulls the 1form $\pi \theta^{c}$ back to zero. The $\pi$-projection of this lift defines a smooth map $j: L \rightarrow \mathbf{E}^{3}$. The Lagrangian Gauss image of this latter map can be geometrically constructed from the lift. In this case we say that $j: L \rightarrow \mathbf{E}^{3}$ is generated by $k: L \rightarrow T^{*} M$ via the contact collar on $i: M \rightarrow \mathbf{E}^{3}$. (For details see the Appendix.) 


\section{THE INVARIANTS}

If $\tilde{j}_{\mu}: L \times[0,1] \rightarrow T^{*} S^{2 *}$ is a homotopy through $\rho \theta^{c}$-exact Lagrangian immersions, then there exists a homotopy $j_{\mu}: L \times[0,1] \rightarrow \mathbf{E}^{3}$ through smooth maps with well-defined Gauss maps $G_{\mu}=\rho \circ \tilde{j}_{\mu}$. We will say that $\tilde{j}_{\mu}$ is an EL-homotopy. (For example, the sphere in Figure 4 of the Appendix has Gauss image a section of $\rho: T^{*} S^{2 *} \rightarrow S^{2 *}$. Thus, its Lagrangian Gauss image is $E L$ homotopic to that of the standard round sphere in $\mathbf{E}^{3}$.) Given a Lagrangian immersion $\tilde{j}: L \rightarrow T^{*} S^{2 *}$ which is the Gauss image of $j: L \rightarrow \mathbf{E}^{3}$, let $C_{\rho} \subset L$ denote the locus where the differential of the Gauss map $G=\rho \circ \tilde{j}$ has rank less than 2, and let $C_{j} \subset L$ denote the locus where $j: L \rightarrow \mathbf{E}^{3}$ has rank less than 2. We will now discuss several invariants of the Lagrangian Gauss image.

(a) The $G$-degree. The degree of the composite $G=\rho \circ \tilde{j}$ is denoted by $\pm G$ degree $\in \mathbf{Z}$ and is defined up to sign. Recall that a global smooth normal vector field $\eta: L \rightarrow S^{2 *}$ induces an area 2 -form $d A(\eta)=\langle\eta \times-,-\rangle$, which is defined on $L-C_{j}$, and (in general) fails to define a global orientation on $L$.

So if we fix an orientation on $L$, then we may decompose $L-C$ as $L^{+} \cup L^{-}$. The $d A(\eta)$-orientation agrees (resp. disagrees) with the fixed orientation on $L^{+}$ (resp. on $L^{-}$). If $n \in S^{2 *}$ is a regular value for $G$ then we denote the number of $G$-preimages by $S^{\#}(n)$, the sheet number at $n$. (For example, the sphere in Figure 2 of the Appendix has $L-C_{j}$ as a pair of disks with $G$-degree 0 , whereas the sphere in Figure 5 has $L-C_{j}$ as a pair of disks with $G$-degree 2.) Recall that in general $C_{j}$ can have nonzero measure in $L$ (see Appendix, (II)(b)(i)).

Theorem 1. If $j: L \rightarrow \mathbf{E}^{3}$ is such that $C_{j}$ has measure zero in $L$ then:

(i) $\pm 4 \pi(G$-degree $)=\int_{L-C_{j}} K d A(\eta)$.

(ii) $\int_{L^{+}}|K| d A(\eta)-\int_{L^{-}}|K| d A(\eta) \geq \min S^{\#}(n)$.

(iii) (Gauss-Bonnett) If $j$ is an immersion then \pm -degree $=\chi(L) / 2$ and for all regular values $n \in S^{2 *}, S^{\#}(n) \geq 1+\operatorname{genus}(L)$.

(iv) If the Lagrangian Gauss image of $j: L \rightarrow \mathbf{E}^{3}$ is generated by $k: L \rightarrow$ $T^{*} M$ via the contact collar on $i: M \rightarrow \mathbf{E}^{3}$ then $G$-degree $= \pm \delta \chi(M) / 2$, where $\delta$ is the degree of the composite $k: L \rightarrow T^{*} M \rightarrow M$.

Proof. On $L-C_{j}$ the pull back 2-form $\eta^{*} d S$ agrees with $K d A(\eta) . K$ and $d A(\eta)$ are not defined on $C_{j}$. Since $\pm 4 \pi(G$-degree) agrees with the integral of $\eta^{*} d S$ over $L$ we have part (i). For part (ii) one need only note that the lefthand side agrees with the integral of $S^{\#}(n)$ over $S^{2 *}$. Part (iii) is classic. For part (iv) consider the homotopy $k_{\mu}: L \rightarrow T^{*} M, \mu \in[0,1]$, defined by $\mu k(x)$, where multiplication is in the fiber. The map $k_{0}$ has image in the zero section of $T^{*} M$ and fails to be an immersion. Because $k_{\mu}$ is exact we have associated functions $s_{\mu}: L \rightarrow \mathbf{R}, \mu \in[0,1]$, and hence lifts to $\operatorname{AFF}\left(X_{\eta}\right)$ as defined in the Appendix. This in turn defines maps $j_{\mu}: L \rightarrow \mathbf{E}^{3}$ with Lagrangian Gauss images $\tilde{j}: L \rightarrow T^{*} S^{2 *}$ and Gauss maps $G_{\mu}: L \rightarrow S^{2 *}$ for all $\mu \in[0,1]$. We need only compute the degree of $G_{0}$. But since $s_{0}$ is the zero function, we have that $j_{0}=i \circ \pi \circ k$, where $\pi \circ k$ is the composite $k: L \rightarrow T^{*} M \rightarrow M$. It follows that the Gauss map of $j_{0}$ is given by $G_{0}=G \circ \pi \circ k$, where $G$ is the Gauss map of the fixed embedding $i: M \rightarrow \mathbf{E}^{3}$. In other words, the Gauss map $G_{0}$ factors through the Gauss map of the embedding via the map $\pi \circ k$. The proof is complete. 
Remarks. Notice that if $j: L \rightarrow \mathbf{E}^{3}$ is generated by $k: L \rightarrow T^{*} \mathbf{E}^{2}$ via a contact collar on a hyperplane $i: \mathbf{E}^{2} \rightarrow \mathbf{E}^{3}$ then the argument of part (iv) above implies that the $G$-degree of $j$ is 0 . We note in passing that the self-intersection number of $\tilde{j}: L \rightarrow T^{*} S^{2 *}$ is $2(G \text {-degree })^{2}$. Thus, if $j: L \rightarrow \mathbf{E}^{3}$ is an immersion then this self-intersection number is $\frac{1}{2}(\chi(M))^{2}$.

(b) The Gauss-Maslov class. Recall that at every point of $T^{*} M$ we have the Grassmann of oriented Lagrangian 2-planes which lie in the tangent space to $T^{*} M$. This Grassmann fibers over a circle (see [GS, A]). Now at every point of $T^{*} M$ we have the tangent space to the fiber which is Lagrangian. It follows that this principal circle bundle over $T^{*} M$ admits a global section and hence is trivial. This yields a map $L G_{2} T\left(T^{*} M\right) \rightarrow S^{1}$, and hence, via a Lagrangian immersion, $k: L \rightarrow T^{*} M$, a cohomology class on $L$. This defines the Maslov class [m] of $k$. In the special case of the Lagrangian Gauss image $\tilde{j}: L \rightarrow T^{*} S^{2 *}$, we refer to this class as $[G m]$, the Gauss-Maslov class. Given $k: L \rightarrow T^{*} M$, its Maslov class can be geometrically computed by way of $\operatorname{graph}(k): L \rightarrow M \times \mathbf{R}$ and intersection with subcotangent bundles $T^{*} S^{1} \rightarrow T^{*} M$ (see [K1]). (For example, the profile curves for the tori in Figures 7 and 8 of the Appendix correspond to such an intersection. For these tori the Maslov class is carried by a standard generator for $H^{1}\left(T^{2}, \mathbf{Z}\right)$, and the period is determined by the number of cusp pairs in the profile curve.) Formally the Maslov class is computed by choosing a horizontal Lagrangian distribution and an adapted Hermitian structure on $T\left(T^{*} M\right)$. The tangent spaces to $L$ then correspond to unitary vector bundle endomorphisms of $T\left(T^{*} M\right)$ over $L$ and the complex determinant models the map $L G_{2} T\left(T^{*} M\right) \rightarrow S^{1}$.

Theorem 2. If the Lagrangian Gauss image of $j: L \rightarrow \mathbf{E}^{3}$ is generated by $k: L \rightarrow T^{*} M$ via the contact collar on $i: M \rightarrow \mathbf{E}^{3}$ then the Maslov class $[m]$ for $k: L \rightarrow T^{*} M$ agrees with the Gauss-Maslov class [Gm] for $\tilde{j}: L \rightarrow T^{*} S^{2 *}$. Furthermore, if $j$ is an immersion then the Gauss-Maslov class is trivial.

Proof. For the first part we need only construct a conformal symplectic vector bundle isomorphism $\Phi$ between the pull back 4-plane bundles $k^{*} T\left(T^{*} M\right)$ and $\tilde{j}^{*} T\left(T^{*} S^{2 *}\right)$ over $L$ which induces an isomorphism between the Lagrangian 2plane subbundles $k_{*}(T L)$ and $\tilde{j}_{*}(T L)$, and which also maps the tangent spaces to $T^{*} M$-fibers to a Lagrangian distribution transverse to the $T^{*} S^{2 *}$-fibers. We now assume familiarity with part (II)(a) of the Appendix.

Now, given $k: L \rightarrow T^{*} M$, we get $k \times(-s): L \rightarrow T^{*} M \times(-\varepsilon, \varepsilon) \simeq \operatorname{AFF}\left(X_{\eta}\right)$ over $V$, which is tangent to the symplectic 4-plane bundle ANN over $L$. This ANN is the 4-plane distribution on $\operatorname{AFF}\left(X_{\eta}\right) \subset T^{*} \mathbf{E}^{3}$ which is annihilated by $i_{\mathrm{sc}}^{*}\left(\pi \theta^{c}\right)+d t$. It inherits a natural symplectic structure from $i_{\mathrm{sc}}^{*} d\left(\pi \theta^{c}\right)$. Recall that $X^{\text {dil }}$ is the characteristic vector field for the 1 -form $\pi \theta^{c}$ and is transverse to $\operatorname{AFF}\left(X_{\eta}\right)$. Now construct the dil-cone on $k(L)$ and intersect it with $\rho^{-1}\left(S^{2 *}\right)$ to get the conormal bundle of $j=\pi \circ \tilde{\eta}$. Projecting $\tilde{\eta}(L) \subset \rho^{-1}\left(S^{2 *}\right)$ along the trajectories of $X^{\text {nor }}$ yields $\tilde{j}$, the Lagrangian Gauss image of the given map $j$. This correspondence maps $k(L) \subset T^{*} M$ to $\tilde{j}(L) \subset T^{*} S^{2 *}$. We claim that this correspondence extends to define the desired vector bundle map $\Phi$.

First $T\left(T^{*} M\right)$ along $k(L)$ can be symplectically identified with ANN over the image of $k \times(-s)$ (since $\psi_{t}^{\rho \eta}$ is a contact-flow on $\operatorname{AFF}\left(X_{\eta}\right)$ ). Next ANN 
over the image of $k \times(-s)$ in $\operatorname{AFF}\left(X_{\eta}\right)$ can be conformally identified with the annihilator of $\pi \theta^{c}$ in $T\left(\rho^{-1}\left(S^{2 *}\right)\right)$ over $\tilde{\eta}(L)$ by projecting along the trajectories of $\psi_{t}^{\text {dil }}$ (since $\psi_{t}^{\text {dil }}$ is a conformal symplectomorphic flow). Finally, this 4-plane distribution can be symplectically identified with $T\left(T^{*} S^{2 *}\right)$ over $\tilde{j}(L)$ by projecting along the trajectories of $\psi_{t}^{\text {nor }}$ (since $\psi_{t}^{\text {nor }}$ is a symplectomorphic flow). The composite of these maps is the desired vector bundle isomorphism $\Phi$. It should be clear to the reader that tangent spaces to $T^{*} M$-fibers are carried by $\Phi$ to a Lagrangian distribution transverse to the $T^{*} S^{2 *}$-fibers, and we have the first part.

For the second part we need only note that given an immersion $i: M \rightarrow \mathbf{E}^{3}$ we may identify $i(M)$ with the zero section $z: M \rightarrow T^{*} M$. In other words, the immersion is generated by $z: M \rightarrow T^{*} M$ via the contact collar on $i: M \rightarrow \mathbf{E}^{3}$, which completes the proof.

(c) The polarization index. Here we assume that the Gauss map $G=\rho \circ \tilde{j}$ of $\tilde{j}: L \rightarrow \mathbf{E}^{3}$ satisfies the following transversality conditions: The differential of the Gauss map has rank $\geq 1$ with differential $G_{*}$ transverse to $\Sigma_{1}$ and $\Sigma_{1,1}$ in $J^{2}\left(L, S^{2 *}\right)$ (see [GG, B]). Thus the locus $C_{\rho}$ where $\rho \circ \tilde{j}$ has rank 1 , is a disjoint union of embedded closed curves marked by a finite number of cusp points $\{\mathrm{cp}\} \subset C_{\rho}$. At these cups points $G$ restricted to $C_{\rho}$ is not immersive. However, the corresponding image curves $G\left(C_{\rho}\right)$ in $S^{2 *}$ have well-defined limiting tangent directions at the cusp points. Now choosing $n \in\left\{S^{2 *}-G\left(C_{\rho}\right)\right\}$ we get the stereographic projection map st: $S^{2 *}-\{n\} \rightarrow \mathbf{E}^{2}$, and hence a welldefined $C_{\rho}$-Gauss map $g: C_{\rho} \rightarrow \mathbf{R P}^{1}$ for the curve st $\circ G: C_{\rho} \rightarrow \mathbf{E}^{2}$. We orient the $C_{\rho}$ so that in $\mathbf{E}^{2}$ the double covered side of $G\left(C_{p}\right)$ lies counterclockwise to the tangent of $G\left(C_{p}\right)$ (see [K1]). The polarization index, $\mathrm{PI}(n)$, is defined to be the degree of the $C_{\rho}$-Gauss map $g$.

Theorem 3. If the Lagrangian Gauss image of $j: L \rightarrow \mathbf{E}^{3}$ satisfies the above conditions then the following hold for all regular values $n \in S^{2 *}$ :

(i) $\operatorname{PI}(n)+2 S^{\#}(n)=\chi(L)$.

(ii) $\operatorname{PI}(n) \leq \chi(L)-2 \mid G$-degree $\mid$.

(iii) If $j$ is an immersion then $\operatorname{PI}(n) \leq-4 \operatorname{genus}(L)$.

Proof. If we delete a small uniformly covered neighborhood $U$ of $n$ in $S^{2 *}$ then $\rho: T^{*}\left(S^{2 *}-U\right) \rightarrow S^{2 *}-U$ can be identified (via stereograph projection) with $\rho: T^{*} B \rightarrow B$, where $B$ is a large open disk in $\mathbf{E}^{2}$ centered at the origin. It follows that $\tilde{j}(L) \cap T^{*}\left(S^{2 *}-U\right)$ can be identified with a Lagrangian $\rho \theta^{c}$ exact immersion $\tilde{j}: L-\bigcup D^{2} \rightarrow T^{*} B$, where the number of disks $D^{2}$ deleted from $L$ agrees with $S^{\#}(n)$. Next $j^{*} \rho \theta^{c}=d s$, where $s: L-U D^{2} \rightarrow \mathbf{R}$. This defines $\operatorname{graph}(\tilde{j}): L-\bigcup D^{2} \rightarrow B \times \mathbf{R}$, where $\operatorname{graph}(\tilde{j})=(\pi \circ \tilde{\eta}, s)$. Over the complement of a smaller open ball $B-B^{\prime}$, the set $\operatorname{graph}(\tilde{j})(L)$ agrees with the graphs of a collection of smooth functions (i.e., $\operatorname{graph}(\tilde{j})(L) \cap\left[\left(B-B^{\prime}\right) \times \mathbf{R}\right]=$ $\bigcup\left[\right.$ graph $\left.\left.f_{i}\left(B^{\prime}\right)\right], i=1, \ldots, S^{\#}(n), f_{i}: B-B^{\prime} \rightarrow \mathbf{R}\right)$. Now we smoothly attach $S^{\#}(n)$-edge disks to the graphs of the $f_{i}$ as in Figure 1. Adjoining this modification to $\operatorname{graph}(\tilde{j})$ over $B$ yields a graph of a multivalued function on $B$ with no periods and hence an exact Lagrangian immersion $L \rightarrow T^{*} B \rightarrow B$. Next, choose a regular value for the $C_{\rho}$-Gauss map $g: C_{\rho} \rightarrow \mathbf{R P}^{1}$. This yields a linear function $l: \mathbf{E}^{2} \rightarrow \mathbf{E}$. Now pull back this linear function over 

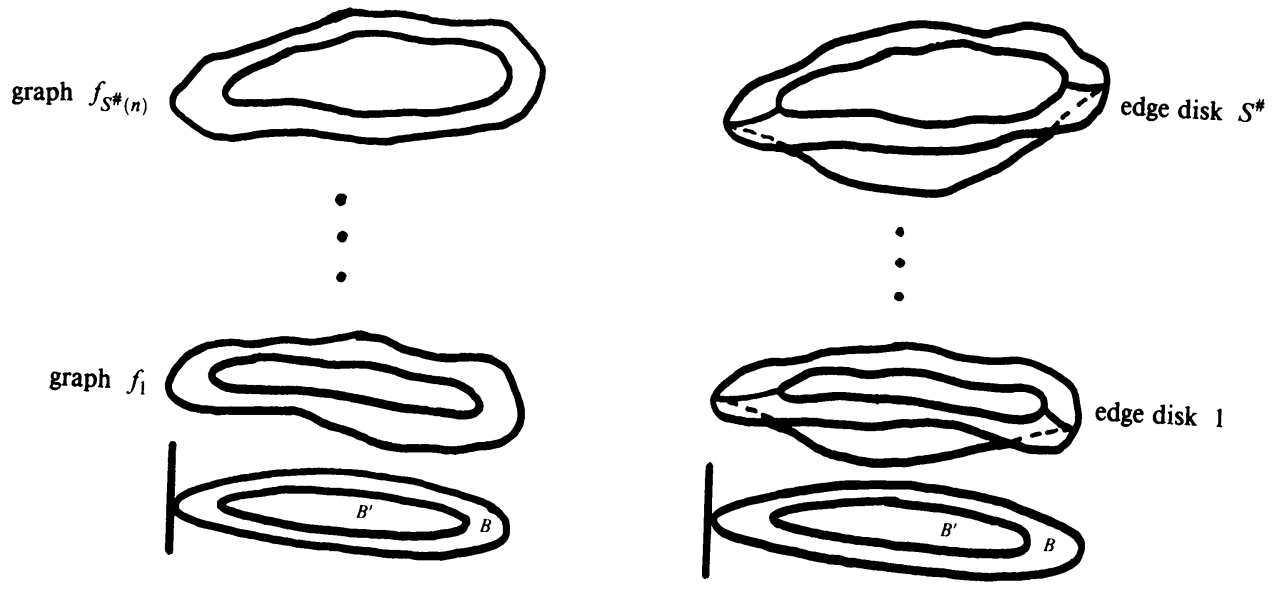

Figure 1

$L \rightarrow T^{*} B \rightarrow B$. Because of the round edge-disks over $B-B^{\prime}$ we have a pair of index 1 critical points on each attached disk. Since $\operatorname{PI}(n)$ agrees with the sum of the indices at critical points in $\rho^{-1}\left(B^{\prime}\right)$, we have part (i). Part (ii) then follows since $S^{\#}(n) \geq \mid G$-degree $\mid$. Part (iii) follows from part (ii) since $S^{\#}(n) \geq[1+\operatorname{genus}(L)]$ for any immersion. We are finished.

Theorem 4. Given a compact orientable surface $L, \alpha \in \mathbf{Z}^{+}$, and $[\tau] \in H^{1}(L, \mathbf{Z})$, there exists a smooth map $j: L \rightarrow \mathbf{E}^{3}$ with immersed Lagrangian Gauss image $\tilde{j}: L \rightarrow T^{*} S^{2 *}$, such that $\mid G$-degree $\mid=\alpha$ and $[\mathrm{Gm}]=[\tau]$.

Proof. By Theorem 1 of $[\mathrm{K} 1]$ we know that there exists a $\pi \theta^{c}$-exact immersion $k: L \rightarrow T^{*} \mathbf{E}^{2}$ with Maslov class $[m]$ agreeing with the given $[\tau]$. Now use the contact collar on a hyperplane embedding $i: \mathbf{E}^{2} \rightarrow \mathbf{E}^{3}$ and Theorem 2 to yield a smooth map $j^{\prime}: L \rightarrow \mathbf{E}^{3}$, with Gauss-Maslov class given by $[\tau]$ and $G$-degree 0 . Now we need only take the connected sum of $j^{\prime}(L)$ and $\alpha$-copies of the 2-sphere given in example (II)(c) Appendix (see Figure 5 there) to get a modified map $j: L \rightarrow \mathbf{E}^{3}$ with $G$-degree agreeing with $\alpha$. We are finished.

Remarks. We note that by deforming the image, of the above generating Lagrangian immersion $k: L \rightarrow T^{*} \mathbf{E}^{2}$, closer to the zero section we shrink the total area (counted with multiplicity) of the $S^{2 *}$-valued Gauss image of $j^{\prime}: L \rightarrow \mathbf{E}^{3}$. It follows that the map $j: L \rightarrow \mathbf{E}^{3}$ constructed in Theorem 4 can be adjusted to satisfy the conditions of Theorem 4 , and

$$
\int_{L^{+}}|K| d A(\eta)-\int_{L^{-}}|K| d A(\eta)=\mid G \text {-degree } \mid+\varepsilon
$$

for any $\varepsilon>0$ sufficiently small.

We also note that Theorem 4 could be proved by constructing the graph of the Lagrangian Gauss image in $S^{2 *} \times \mathbf{R}$ (using Theorem 1 of [K1]) and then applying the lemma in the Appendix. This approach has the advantage that it could be used to control the image of the singular locus $G\left(C_{\rho}\right)$ in $S^{2 *}$. In particular, one could construct $j: L \rightarrow \mathbf{E}^{3}$ as above with $G\left(C_{\rho}\right)$ simple. 


\section{Applications}

The simplest application of the above construction is to identify restrictions on the $G$-image of $C_{\rho}$ in $S^{2 *}$. For example, an embedded pair of concentric simple closed curves in $S^{2 *}$ cannot occur as the image of $G\left(C_{\rho}\right)$ for a surface of positive genus since, regardless of orientation, there would be an $n \in S^{2 *}$ for which $\operatorname{PI}(n) \geq 0$. See [K2] for additional restrictions.

We can also use the above to find homotopy obstructions for solutions to spherical Monge-Ampere PDE. A differential ideal generated by one smooth 2form $(\Omega)$ on $\rho^{-1}\left(S^{2 *}\right)=\left(T^{*} S^{2 *} \times \mathbf{R}, \pi \theta^{c}\right)$ defines a PDE for maps $j: L \rightarrow \mathbf{E}^{3}$ via the condition that $\tilde{\eta}: L \rightarrow \rho^{-1}\left(S^{2 *}\right)$ annihilate both $\pi \theta^{c}$ and the auxiliary $(\Omega)$. If one chooses local graph coordinates for $j$ and corresponding coordinates on $S^{2 *},(p, q) \rightarrow(p, q, 1) /\left(1+\rho^{2}+q^{2}\right)^{1 / 2}$, then this condition will be locally represented by a classical Monge-Ampère type PDE (see [K3]). The construction of symplectic and cotangent characteristic varieties, as discussed in [K3], adapt directly to this setting. It follows that if:

(i) $\Omega$ is elliptic then the [Gm]-class must be trivial on any solution;

(ii) $\Omega$ is elliptic and the cotangent characteristic variety is uniformly degenerate then $\pm G$-degree $=S^{\#}(n)$ on any solution;

(iii) $\Omega$ is hyperbolic with cotangent characteristic variety nondegenerate then for all $n \in S^{2 *}$ the polarization index, $\operatorname{PI}(n)$, is carried by a cohomology class on $L-G^{-1}(n)$.

The proofs are direct adaptations of the arguments in [K3]. As applications of the above we need only note that many of the classical PDE for surfaces in $\mathbf{E}^{3}$ define spherical Monge-Ampère PDE. For example, if $j: L \rightarrow \mathbf{E}^{3}$ has constant mean curvature at every immersive point then $\tilde{j}: L \rightarrow T^{*} S^{2 *}$ annihilates a global elliptic differential ideal. Hence, the Gauss-Maslov class must be trivial. Similarly, if $j: L \rightarrow \mathbf{E}^{3}$ has constant negative Gauss curvature at every immersive point then $\tilde{j}: L \rightarrow T^{*} S^{2 *}$ annihilates a global hyperbolic nondegenerate differential ideal. Hence, the polarization index is carried by a cohomology class as in (iii).

\section{APPENDIX}

(I) The Lagrangian Gauss image and related constructions. Let $\pi: T^{*} \mathbf{E}^{3} \rightarrow \mathbf{E}^{3}$ denote the cotangent bundle of $\mathbf{E}^{3}$ with canonical projection $\pi$ and canonical 1 -form $\pi \theta^{c}$. Since $\mathbf{E}^{3}$ carries a flat connection, we have a horizontal projection $\rho: T^{*} \mathbf{E}^{3} \rightarrow \mathbf{E}^{3 *}$ which is defined by parallel translation. Via double dualization we have an associated horizontal canonical 1 -form $\rho \theta^{c}$. Thus we may write $T^{*} \mathbf{E}^{3}=\mathbf{E}^{3}+\mathbf{E}^{3 *}$ with $\pi$ and $\rho$ the respective projections onto summands, so that $d\left(\pi \theta^{c}\right)=-d\left(\rho \theta^{c}\right)$ defines the canonical symplectic structure $\omega$ on $T^{*} \mathbf{E}^{3}$. A typical element of $T^{*} \mathbf{E}^{3}$ will be denoted by $\left\langle v_{x},\right\rangle: T_{x} \mathbf{E}^{3} \rightarrow \mathbf{R}$, where $v_{x} \in T_{x} \mathbf{E}^{3}$. The Legendre symplectomorphism Leg: $T^{*} \mathbf{E}^{3} \rightarrow T^{*} \mathbf{E}^{3}$, defined by $\operatorname{Leg}\left(\left\langle v_{x},-\right\rangle\right)=-\left(\left\langle x_{v},-\right\rangle\right)$, interchanges vertical and horizontal projections. Furthermore, $\pi \theta^{c}=\operatorname{Leg}^{*}\left(-\rho \theta^{c}\right)$. The inner product $\langle$,$\rangle induces the dual$ norm \|\|$_{*}: \mathbf{E}^{3 *} \rightarrow \mathbf{R}$. This yields a pull back function $\rho^{*}\left(\|\|_{*}\right): T^{*} \mathbf{E}^{3} \rightarrow \mathbf{R}$ with Hamiltonian vector field $X^{\text {nor }}$ and flow $\psi_{t}^{\text {nor }}$. The inner product also induces a diagonal inner product function dip: $T^{*} \mathbf{E}^{3} \rightarrow \mathbf{R}$, defined by $\operatorname{dip}\left(\left\langle v_{x},-\right\rangle\right)=$ 
$\langle v, x\rangle$. Notice that the set dip $=0$ in $T^{*} \mathbf{E}^{3}$ is preserved by Leg. The R-

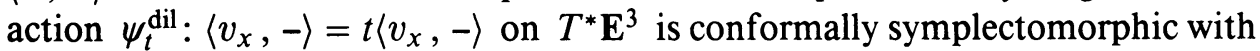
generating vector field denoted by $X^{\text {dil }}$.

(a) The conormal subbundle. Now let $i: M \rightarrow \mathbf{E}^{3}$ be a smoothly immersed, compact, oriented surface with unit normal vector field $\eta$ and associated Gauss map $G: M \rightarrow S^{2}$. Given such a surface we get the conormal subbundle of $T^{*} \mathbf{E}^{3}$ over $i(M)$,

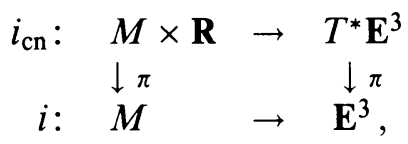

where $i_{\mathrm{cn}}(m, s)=s\langle\eta(m),-\rangle \in T^{*} \mathbf{E}^{3}$ for $m \in M$ and $s \in \mathbf{R}$. This is an immersed Lagrangian 3-fold in $T^{*} \mathbf{E}^{3}$ which annihilates $\pi \theta^{c}$ (i.e., $i_{\mathrm{cn}}^{*} \omega=0=$ $\left.i^{*}\left(\pi \theta^{c}\right)\right)$. Notice that $i_{\mathrm{cn}}(m, 0)=i(m)$ for all $m \in M$, so that intersecting the $i_{\mathrm{cn}}$-image with the $\pi$-zero section reproduces the given immersed surface.

(b) The subcotangent bundle. Given an immersed surface we also get a subcotangent bundle of $T^{*} \mathbf{E}^{3}$ over $i(M)$,

$$
\begin{array}{cccc}
i_{\mathrm{sc}}: & T^{*} M & \rightarrow & T^{*} \mathbf{E}^{3} \\
& \downarrow \pi & & \downarrow \pi \pi \\
i: & M & \rightarrow & \mathbf{E}^{3},
\end{array}
$$

where a covector $l: T_{m} M \rightarrow \mathbf{R}$ at $m \in M$ is assigned the ambient covector $l^{a}: T_{i(m)} \mathbf{E}^{3} \rightarrow \mathbf{R}$ at $i(m)$ such that $l^{a}(\eta(m))=0$ and $l^{a}=l$ on $T_{m} M$. That is, $i_{\mathrm{sc}}\left(l_{m}\right)=l_{i(m)}^{a}$. By its very definition the 1 -form $\pi \theta^{c}$ on $T^{*} \mathbf{E}^{3}$ pulls back over $i_{\mathrm{sc}}$ to the canonical 1-form on $\pi: T^{*} M \rightarrow M$. Hence, $i_{\mathrm{sc}}$ is a symplectic immersion.

(c) The contact structure of $\rho^{-1}\left(S^{2 *}\right)$. Now consider the round unit sphere $S^{2 *}$ at the origin in $\mathbf{E}^{3 *}$. The $\rho$-preimage $\rho^{-1}\left(S^{2 *}\right)$ is a level set of the Hamiltonian function $\rho^{*}\left(\|\|_{*}\right): T^{*} \mathbf{E}^{3} \rightarrow \mathbf{R}$. Now for any point $\left\langle v_{0},-\right\rangle$ in $S^{2 *}$, the $\rho$-fiber $\rho^{-1}\left(\left\langle v_{0},-\right\rangle\right)$ is a horizontal $\mathbf{E}^{3}$ to which the Hamiltonian vector field $X^{\text {nor }}$ is tangent. Furthermore, the $\pi_{*}$-projection of $X^{\text {nor }}$, restricted to $\rho^{-1}\left(\left\langle v_{0},-\right\rangle\right)$, is parallel to $v_{0} \in T_{0} \mathbf{E}^{3}$. Consider the 2-plane subbundle of $\rho^{-1}\left(S^{2 *}\right)$ whose fiber at $\left\langle v_{0},-\right\rangle$ is the subspace in $\rho^{-1}\left(\left\langle v_{0},-\right\rangle\right)$ perpendicular to $v_{0}$. Since the flow $\psi_{t}^{\text {nor }}$ preserves each $\rho$-fiber, this 2-plane bundle is a global transverse section for $\psi_{t}^{\text {nor }}$ on $\rho^{-1}\left(S^{2 *}\right)$. We claim this vector bundle is symplectomorphic to the cotangent bundle of the sphere. (It will be denoted by $h: T^{*} S^{2 *} \rightarrow T^{*} \mathbf{E}^{3}$, with projection $\rho: T^{*} S^{2 *} \rightarrow S^{2 *}$.) To see this, first notice that this 2-plane bundle lies in $\operatorname{dip}=0$ and recall that dip $=0$ is preserved by the Legendre symplectomorphism. Now if we take $i: S^{2} \rightarrow \mathbf{E}^{3}$ to be the round sphere at the origin, and observe that the associated subcotangent bundle $i_{\mathrm{sc}}: T^{*} S^{2} \rightarrow T^{*} \mathbf{E}^{3}$ lies in $\operatorname{dip}=0$, then we see that Leg transforms $i_{\mathrm{sc}}\left(T^{*} S^{2}\right)$ into $\rho^{-1}\left(S^{2 *}\right)$, preserving the linear structure of the fibers and $\omega$. The canonical 1-form $\pi \theta^{c}$ is transformed to $-\rho \theta^{c}$. Since, as a subset of $T^{*} \mathbf{E}^{3}$, this 2-plane bundle agrees with $\{$ dip $=0\} \cap\left\{\rho^{*}\left(\|\|_{*}\right)=1\right\}$, we have our claim. Notice that, on $\rho^{-1}\left(S^{2 *}\right)$, we have the Lie derivatives $L_{X} \operatorname{nor}\left(\pi \theta^{c}\right)=0$ and $\pi \theta^{c}\left(X^{\text {nor }}\right)=1$. Thus, $\rho^{-1}\left(S^{2 *}\right)$ with the 1 -form $\pi \theta^{c}$ is a contact manifold with canonical vector field $X^{\text {nor }}$ and global transverse section $h\left(T^{*} S^{2 *}\right) \subset \rho^{-1}\left(S^{2 *}\right)$ (i.e., contact equivalent to $\left(T^{*} S^{2 *}\right) \times \mathbf{R}$ with 1-form $\left.h^{*}\left(\pi \theta^{c}\right)+d t\right)$. 
(d) The Lagrangian Gauss image. We now define the Lagrangian Gauss image of an immersion $i: M \rightarrow \mathbf{E}^{3}$ as follows:

First, intersect the conormal bundle $i_{\mathrm{cn}}(M \times \mathbf{R})$ with the preimage $\rho^{-1}\left(S^{2 *}\right)$. Since $X^{\text {dil }}$ is tangent to the conormal bundle, this intersection is transverse and is a section of $\pi: M \times \mathbf{R} \rightarrow M$. Thus, it defines an immersion $\tilde{\eta}: M \rightarrow$ $\rho^{-1}\left(S^{2 *}\right) \subset T^{*} \mathbf{E}^{3}$, such that $\tilde{\eta}^{*}\left(\pi \theta^{c}\right)=0$. Notice that $\tilde{\eta}(m)$ can be written as $\langle\eta(m),-\rangle \in T_{i(m)}^{*} \mathbf{E}^{3}$, where $\eta$ is the unit normal for the given immersion. It follows that $\pi \circ \tilde{\eta}=i$, and that $\pi_{*} X^{\text {nor }}$ evaluated at $\tilde{\eta}(m)$ is parallel to $\eta(m)$. Hence, $X^{\text {nor }}$ is not tangent to $\tilde{\eta}(M)$ in $\rho^{-1}\left(S^{2 *}\right)$.

Second, consider the immersed 3-fold $\psi_{t}^{\text {nor }} \circ \tilde{\eta}: M \times \mathbf{R} \rightarrow \rho^{-1}\left(S^{2 *}\right) \subset T^{*} \mathbf{E}^{3}$ defined by $\psi_{t}^{\text {nor }}(\tilde{\eta}(m)), m \in M, t \in \mathbf{R}$. Since $\tilde{\eta}^{*} \omega=d \tilde{\eta}^{*}\left(\pi \theta^{c}\right)=0$, this is a Lagrangian solution to the Hamilton-Jacobi PDE $\rho^{*}\left(\|\|_{*}\right)=1$. Recall that $h\left(T^{*} S^{2 *}\right)$ is a global transverse section for the flow of $\psi_{t}^{\text {nor }}$ on $\rho^{-1}\left(S^{2 *}\right)$. Thus, the intersection of $\psi_{t}^{\text {nor }} \circ \tilde{\eta}(M \times \mathbf{R})$ and $T^{*} S^{2 *} \cong h\left(T^{*} S^{2}\right)$ in $\rho^{-1}\left(S^{2 *}\right)$ is a smooth Lagrangian immersion of $M$ which we denote $\tilde{i}: M \rightarrow T^{*} S^{2 *}$.

This defines the Lagrangian Gauss image of the given immersion $i: M \rightarrow \mathbf{E}^{3}$. Since $\psi_{t}^{\text {nor }}$ preserves the $\rho$-fibers (i.e., $\rho \circ \psi_{T}^{\text {nor }}=\rho$ ), we can recover the classic Gauss map via $G=\rho \circ \tilde{i}: M \rightarrow S^{2 *}$. The Lagrangian Gauss image of an immersion $i: M \rightarrow \mathbf{E}^{3}$ is $\rho \theta^{c}$-exact (i.e., $\tilde{i}: M \rightarrow T^{*} S^{2 *}$ satisfies $\tilde{i}^{*}\left(\rho \theta^{c}\right)=$ $d f$, where $f: M \rightarrow \mathbf{R})$. To see this recall that $\tilde{\eta}^{*}\left(\pi \theta^{c}\right)=0$ implies that the periods of the 1-form $\left(\psi_{t}^{\text {nor }} \circ \tilde{\eta}\right)^{*}\left(\pi \theta^{c}\right)$ on $M \times \mathbf{R}$ are all zero. Now $d\left(\pi \theta^{c}\right)=$ $\omega=-d\left(\rho \theta^{c}\right)$ implies that $\pi \theta^{c}$ and $-\rho \theta^{c}$ differ by the exterior derivative of a function on $T^{*} \mathbf{E}^{3}$. Thus, the $\pi \theta^{c}$-periods on the $\rho \theta^{c}$-periods agree on $M \times \mathbf{R}$. We now show that this correspondence is essentially reversible.

Lemma. Given a smoothly immersed, compact, orientable, $\rho \theta^{c}$-exact, Lagrangian immersion $\tilde{j}: L \rightarrow T^{*} S^{2 *}$, there exists a unique smooth 1-parameter family of smooths maps $j_{\lambda}: L \rightarrow \mathbf{E}^{3}, \lambda \in \mathbf{R}$, with Gauss map $G=\rho \circ \tilde{j}$.

Proof. Given $\tilde{j}: L \rightarrow T^{*} S^{2 *} \subset \rho^{-1}\left(S^{2 *}\right), \rho \theta^{c}$-exact, consider the Lagrangian 3-fold $\psi_{t}^{\text {nor }} \circ \tilde{j}: L \times \mathbf{R} \rightarrow \rho^{-1}\left(S^{2 *}\right)$ defined by $\psi_{t}^{\text {nor }}(\tilde{j}(l)), l \in L, t \in \mathbf{R}$. We need only show that there exists a smooth section of $L \times \mathbf{R}, t: L \rightarrow \mathbf{R}$, such that the pull back of $\pi \theta^{c}$ over $\tilde{\eta}: L \rightarrow \rho^{-1}\left(S^{2 *}\right), \tilde{\eta}(l)=\psi_{t(l)}^{\text {nor }}(\tilde{j}(l)), l \in L$, is zero. This is because $\pi \circ \tilde{\eta}: L \rightarrow \mathbf{E}^{3}$ has a natural conormal bundle defined by the union of $\pi$-fiber rays through points of the $\tilde{\eta}$-image (i.e., the image of the map $\left.L \times \mathbf{R} \rightarrow T^{*} \mathbf{E}^{3},(l, \lambda) \rightarrow \lambda \cdot \tilde{\eta}(l), l \in L, \lambda \in \mathbf{R}\right)$, and hence has a Gauss map defined by $G=\rho \circ \tilde{\eta}=\rho \circ \tilde{j}$.

To show that there exist such sections recall that $\rho^{-1}\left(S^{2 *}\right)$ with the 1 -form $\pi \theta^{c}$ is a contact manifold with transverse section $h: T^{*} S^{2 *} \rightarrow T^{*} \mathbf{E}^{3}$. Since $\tilde{j}: L \rightarrow T^{*} S^{2 *}$ is $\rho \theta^{c}$-exact, it is $\pi \theta^{c}$-exact. Thus, $(h \circ \tilde{j})^{*} \pi \theta^{c}=d s, s: L \rightarrow \mathbf{R}$, and $\psi_{\lambda-s}^{\text {nor }}(h \circ j): L \rightarrow \rho^{-1}\left(S^{2 *}\right)$, defined by $\psi_{\lambda-s}^{\text {nor }}(h(\tilde{j}(l))), l \in L, \lambda \in \mathbf{R}$, is the one-parameter family of sections we seek.

(II) Examples.

(a) Contact collars over embedded submanifolds of $\mathbf{E}^{3}$. Given a smoothly embedded orientable surface $i: M \rightarrow \mathbf{E}^{3}$ and a smooth exact Lagrangian immersion $k: L \rightarrow T^{*} M$, whose image lies sufficiently close to the zero section, we will show there exists a smooth map $j: L \rightarrow \mathbf{E}^{3}$ with well-defined immersed 
Lagrangian Gauss image $\tilde{j}: L \rightarrow T^{*} S^{2 *}$. We begin by modifying the subcotangent bundle construction. This modification will be called a contact collar. Let $X_{\eta}$ denote the smooth vector field on $\mathbf{E}^{3}$ which: has support lying in a tubular neighborhood $U$ of $i: M \rightarrow \mathbf{E}^{3}$; agrees with the unit normal along $i(M)$; on a smaller $\varepsilon$-tubular neighborhood $V \subset U$ agrees with unit normal vectors to the surface parallel transported along normal lines. (So that on $V$ the vector field $X_{\eta}$ is of unit length, and tangent to a line normal to $M$.) This vector field has a flow $\Psi_{t}$ on $\mathbf{E}^{3}$ which prolongs to a flow $\psi_{t}^{p \eta}=\left(\Psi_{t}\right)^{*}$ on $T^{*} \mathbf{E}^{3}$ with generator $X^{p \eta}$.

Now let $\pi: G_{2}\left(\mathbf{E}^{3}\right) \rightarrow \mathbf{E}^{3}$ denote the Grassmann bundle whose $\pi$-fiber at $x \in$ $\mathbf{E}^{3}$ is the set of oriented 2-planes in $T_{x} \mathbf{E}^{3}$. It carries the tautological differential ideal $\left(\pi \theta^{c}\right)$, where a vector tangent to $G_{2}\left(\mathbf{E}^{3}\right)$ at a 2-plane is annihilated by $\left(\pi \theta^{c}\right)$ if and only if its $\pi_{*}$-projection lies in the 2-plane. (As a bundle it can be identified with the complement of the zero section in $\pi: T^{*} \mathbf{E}^{3} \rightarrow \mathbf{E}^{3}$ modulo the action of the flow $\psi_{t}^{\text {dil }}$. The differential ideal generated by $\pi \theta^{c}$ on $T^{*} \mathbf{E}^{3}-\{0\}$ then agrees with the tautological ideal.) Since $\psi_{t}^{p \eta}$ is a prolongation, it is a vector bundle automorphism of $T^{*} \mathbf{E}^{3}$ and hence descends to define a flow, also denoted by $\psi_{t}^{p \eta}$, on $G_{2}\left(\mathbf{E}^{3}\right)$.

Now, given an embedding $i: M \rightarrow \mathbf{E}^{3}$, we get

$$
\begin{array}{cccc}
i_{\mathrm{c}}: & T^{*} M & \rightarrow & G_{2}\left(\mathbf{E}^{3}\right) \\
& \downarrow \pi & & \downarrow \pi \\
i: & M & \rightarrow & \mathbf{E}^{3},
\end{array}
$$

where a covector $l: T_{m} M \rightarrow \mathbf{R}$ at $m \in M$ is assigned the subspace annihilated by the ambient covector $l^{a}: T_{i(m)} \mathbf{E}^{3} \rightarrow \mathbf{R}$ at $i(m)$, such that $l^{a}(\eta(m))=1$ and $l=l^{a}$ on $T_{m} M$. Notice that the image of $i_{\mathrm{c}}$ lies in $\operatorname{AFF}\left(X_{\eta}\right)$, the set of covectors at points in $V \subset \mathbf{E}^{3}$ which yield value 1 when paired with $X_{\eta}$. This is an affine 2-plane bundle which is transverse to $X^{\text {dil }}$. Hence, it will serve as an incomplete model for $G_{2}\left(\mathbf{E}^{3}\right)$ over $V$. Under this embedding $\pi \theta^{c}$ is pulled back to $i_{\mathrm{sc}}^{*} \pi \theta^{c}$, the canonical 1-form on $T^{*} M$. Now we compose this map with the prolonged flow to get the contact collar of $i(M)$ over $V \subset \mathbf{E}^{3}$ :

$$
\begin{array}{rcccc}
i_{\mathrm{cc}}=\psi_{t}^{p \eta} \circ i_{\mathrm{c}}: & T^{*} M \times(-\varepsilon, \varepsilon) & \rightarrow & G_{2}\left(\mathbf{E}^{3}\right) \\
& \downarrow & & \downarrow \pi \\
\Psi_{t} \circ i: & M \times(-\varepsilon, \varepsilon) & \rightarrow & V \subset \mathbf{E}^{3} .
\end{array}
$$

The image also lies in $\operatorname{AFF}\left(X_{\eta}\right)$. On $\pi^{-1}(V)$ the pull back $i_{\text {cc }}^{*}\left(\pi \theta^{c}\right)$ takes the form $\left(i_{\mathrm{sc}}^{*} \pi \theta^{c}\right)+d t$.

Now if we are given an exact, compact, Lagrangian immersion $k: L \rightarrow T^{*} M$ such that $\left(i_{\mathrm{sc}} \circ k\right)^{*} \pi \theta^{c}=d s, s: L \rightarrow \mathbf{R},|s|<\varepsilon$, then $k \times(-s): L \rightarrow T^{*} M \times \mathbf{R}$ yields an immersive composite $\tilde{\eta}=\psi_{-s}^{p \eta} \circ k: L \rightarrow \operatorname{AFF}\left(X_{\eta}\right) \subset T^{*} \mathbf{E}^{3}, \tilde{\eta}(l)=$ $\psi_{-s(l)}^{p \eta}(k(l)), l \in L$, which satisfies $(\tilde{\eta})^{*}\left(\pi \theta^{c}\right)=0$. It follows that $j=\pi \circ$ $\tilde{\eta}: L \rightarrow \mathbf{E}^{3}$ has conormal bundle given by the union of $\pi$-fibers through points of the $\tilde{\eta}$-image in $T^{*} \mathbf{E}^{3}$ (i.e., the image of the map $L \times \mathbf{R} \rightarrow T^{*} \mathbf{E}^{3},(l, \lambda) \rightarrow$ $\lambda \cdot \tilde{\eta}(l), l \in L, \lambda \in \mathbf{R})$. We call this the dil-cone on $k(L)$. Hence, this $j$ has a well-defined immersed Lagrangian Gauss image.

For the converse, given a smooth map $j: L \rightarrow \mathbf{E}^{3}$ with well-defined Gauss map $G$ whose image lies in the tubular neighborhood $V$, the tangent plane to $j(L)$ at $l \in L$ is defined to be the kernel of $\left\langle v_{0},-\right\rangle$ in $\mathbf{E}^{3}$ where $G(l)=\left\langle v_{0},-\right\rangle$. If these tangent planes do not contain $X_{\eta}$ then there is an associated Lagrangian map $k: L \rightarrow T^{*} M$. Regularity conditions on $j$ are needed in order for $k$ to be 
immersive (see [A, K1] and example (b)(ii) below). Examples (c) and (d) below will illustrate these constructions. We note in passing that the subcotangent and contact collar construction do not require a Euclidean structure. That is, if $i: M \rightarrow \mathbf{R}^{3}$ is an embedding and $X$ is a vector field on $\mathbf{R}^{3}$ transverse to $M$ then there exist maps as in diagrams (D1)-(D4).

(b) Coordinate models. (i) Constructions of $\S 1$. Let us illustrate the above in terms of local coordinate representatives. If $(x, y, z)$ are orthonormal linear coordinates on $\mathbf{E}^{3}$ then let $(x, y, z, p, q, r)$ be the associated cotangent coordinates on $T^{*} \mathbf{E}^{3}$, so that $\pi \theta^{c}$ is represented by $p d x+q d y+r d z ; \rho \theta^{c}$ is represented by $x d p+y d q+z d r ; \rho^{*}\left(\|\|_{*}\right)$ is represented by $\left(p^{2}+q^{2}+r^{2}\right)^{1 / 2} ; \operatorname{dip}$ is represented by $p x+q y+r z$; and $\psi_{t}^{\text {nor }}$ is represented by $(x+t p, y+t q, z+$ $\operatorname{tr}, p, q, r)$. Note that $L_{X^{\text {nor }}} \pi \theta^{c}=d\left(\rho^{*}\|\|_{*}\right)$ and $\pi \theta^{c}\left(X^{\mathrm{nor}}\right)=\left(\rho^{*}\|\|_{*}\right)^{2}$.

Now if an immersion of an open domain $i: D \subset \mathbf{R}^{2} \rightarrow \mathbf{E}^{3}$ is given by $i(u, v)=(x(u, v), y(u, v), z(u, v))$ with $\mathbf{E}^{3}$-unit normal given by $(p(u, v)$, $q(u, v), r(u, v)),(u, v) \in D \subset \mathbf{R}^{2}$, then $\tilde{i}(u, v)$ is given by $(x-t p, y-t q, z-$ $t r, p, q, r)_{(u, v)}$, where $t(u, v)=p x+q y+r z$. So if $i(u, v)$ parametrizes a piece of the round 2-sphere centered at the origin then $\pi \circ \tilde{i}(u, v)=(0,0)$ and $G(u, v)=\rho \circ \tilde{i}(u, v)=i(u, v)$. Thus, $\pi \circ \tilde{i}$ can be singular while $G(u, v)$ is a diffeomorphism. If a Lagrangian immersion $\tilde{j}: D \rightarrow T^{*} S^{2 *}$ is represented by $\tilde{j}(u, v)=(x, y, z, p, q, r)_{(u, v)}$ with $p^{2}+q^{2}+r^{2}=1, p x+q y+r z=0$, and $\tilde{j}^{*} \omega=0$, then

$$
\left(p x_{u}+q y_{u}+r z_{u}\right) d u+\left(p x_{v}+q y_{v}+r z_{v}\right) d v=d s(u, v)
$$

and $j(u, v)$ is given by $(x-s p, y-s q, z-s r)$. So if $\tilde{j}(u, v)$ parametrizes a piece of the round 2-sphere in $\pi^{-1}(0,0,0)$ then $s$ is constant and $j(u, v)$ parametrizes a piece of a round sphere in $\mathbf{E}^{3}$. Now if $(u, v, a, b)$ are the coordinates on $T^{*} D$ which represent the covector $\langle a \partial u+b \partial v,-\rangle$ then

$$
i_{\mathrm{sc}}(u, v, a, b)=\left(x, y, z, a x_{u}+b x_{v}, a y_{u}+b y_{v}, a z_{u}+b z_{v}\right) \text {. }
$$

Furthermore, if we choose (nonlinear) local coordinates on $\mathbf{E}^{3}$ so that an immersion is represented by $i(u, v)=(u, v, 0)$ and $X_{\eta}$ is represented by $\partial z$ then in associated cotangent coordinates on $T^{*} D$ and $T^{*} \mathbf{E}^{3}$ the contact collar is represented by $i_{\mathrm{cc}}(u, v, a, b, t)=(u, v, t, a, b, 1)$, and $\operatorname{AFF}\left(X_{\eta}\right)$ is represented by the set $r=1$. Since $\pi \theta^{c}$ is represented by $p d x+q d y+r d z$, we have that the pull back $i_{\mathrm{cc}}\left(\pi \theta^{c}\right)$ is represented by $a d u+b d v+d t=i_{\mathrm{sc}}^{*} \pi \theta^{c}+d t$. Notice that in the special case when $k: D \rightarrow T^{*} D$ is the zero section, $k(u, v)=$ $(u, v, 0,0)$, then $\tilde{\eta}=\psi_{-s}^{p \eta} \circ k(u, v)=(u, v, 0,0,0, s)$ and hence $\tilde{\eta}$ reproduces the conormal of the given embedding.

(ii) Nonimmersive maps. The classical examples of smooth nonimmersive germs $j: \mathbf{R}^{2} \rightarrow \mathbf{R}^{3}$ are as follows. Edges: $j(u, v)=\left(u, v^{2}, \int_{0}^{v} 2 v f(u, v)\right)$, with nonzero normal field $(*,-f, 1)$. Here the map $\tilde{j}: \mathbf{R}^{2} \rightarrow T^{*} S^{2 *}$ is immersive at $(0,0)$ if and only if $f_{v}(0,0) \neq 0$. Tails: $j(u, v)=\left(u, v^{3}-\right.$ $\left.u v, \int_{0}^{v}\left(3 v^{2}-u\right) f(u, v)\right)$ with nonzero normal field $(*,-f, 1)$. Here the map $\tilde{j}: \mathbf{R}^{2} \rightarrow T^{*} S^{2 *}$ is immersive at $(0,0)$ if and only if $f_{v}(0,0) \neq 0$. Notice that as one crosses a curve of singular points where the rank of $j_{*}$ is 1 (i.e., $C_{j} \subset \mathbf{R}^{2}$ ) then the orientation induced by the nonzero normal field changes. (From the Arnold viewpoint [A], these maps are $C^{\infty}$-generic, whereas from the 


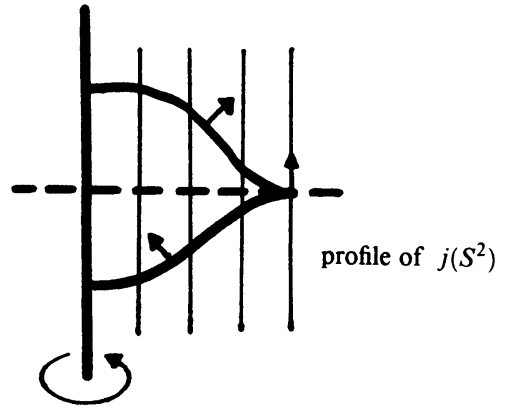

FIGURE 2

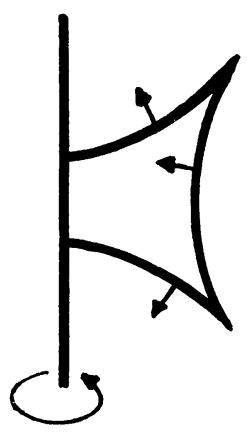

Figure 4

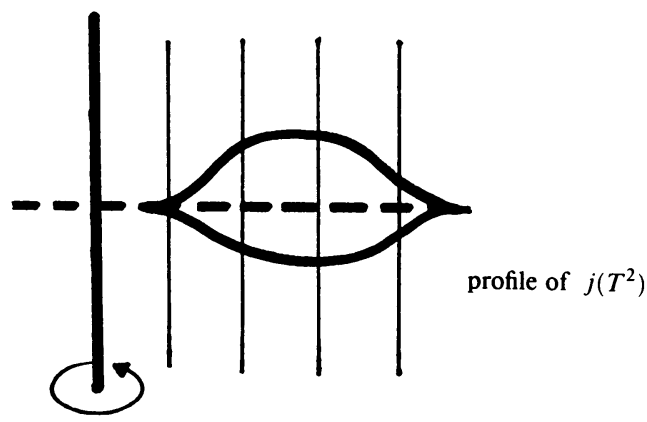

FIGURE 6

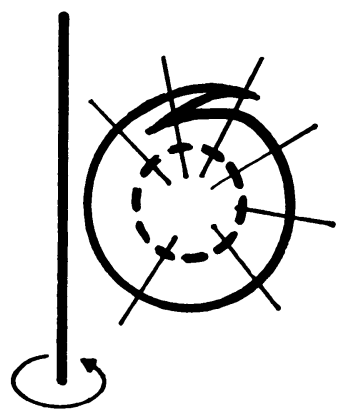

Figure 8

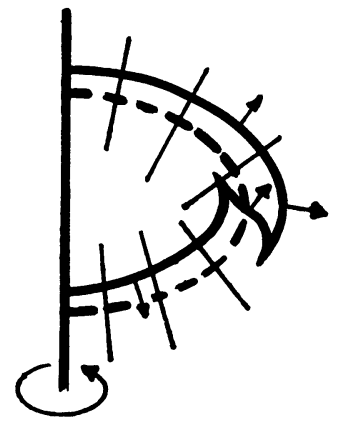

Figure 3

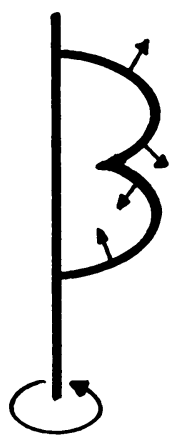

FIGURE 5

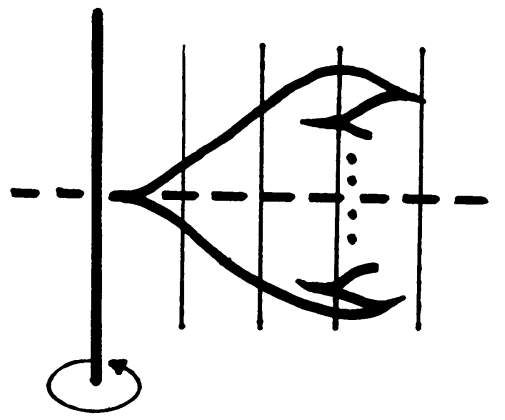

FIGURE 7

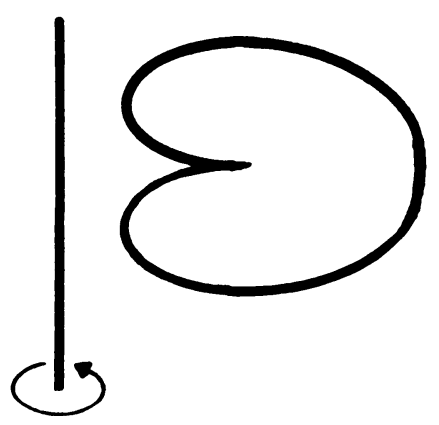

FIGURE 9 
Mond viewpoint $[\mathrm{M}]$, they are not. A simple example of a germ $j: \mathbf{R}^{2} \rightarrow \mathbf{R}^{3}$ without a Gauss map is the Cross Cap $j(u, v)=\left(u, v^{2}, u v\right)$.)

(c) Spheres. Consider the nonimmersive maps of revolution $j: S^{2} \rightarrow \mathbf{E}^{3}$ given by Figures $2-5$. We assume the profile curves are such that, at singular points, $j$ is locally equivalent to the edges of example (b)(ii). The cusp points in the profile curve correspond to the closed edge curves $C_{j} \subset S^{2}$. Notice that upon crossing these curves the orientation induced by the global normal field changes. In Figure 2 the dashed line corresponds to a hyperplane embedding $i: \mathbf{E}^{2} \rightarrow \mathbf{E}^{3}$, and the tangent space to the sphere is everywhere transverse to $X_{\eta}$. It follows that this sphere is generated by a Lagrangian immersion $k: S^{2} \rightarrow T^{*} \mathbf{E}^{2}$ via the contact collar on the hyperplane. Similarly, in Figure 3 the dashed line corresponds to an embedding $i: S^{2} \rightarrow \mathbf{E}^{3}$, and the nonimmersed sphere is generated by a Lagrangian immersion $k: S^{2} \rightarrow T^{*} S^{2}$. If we delete the edge curves in Figures 4 and 5 then the surfaces are locally convex. Thus, for Figure 4 the Gauss map is a diffeomorphism, and in Figure 5 the Gauss map is a local diffeomorphism on the complement of the edge points.

(d) Tori. Consider the nonimmersive maps of tori $j: T^{2} \rightarrow \mathbf{E}^{3}$ given in Figures 6-9. Both of the tori in Figures 6 and 7 are generated by Lagrangian immersions $k: T^{2} \rightarrow T^{*} \mathbf{E}^{2}$ via a contact collar. Figure 8 is generated by $k: T^{2} \rightarrow T^{*} T^{2}$, where $i: T^{2} \rightarrow \mathbf{E}^{3}$ is the standard torus of revolution. In contrast, the map $T^{2} \rightarrow \mathbf{E}^{3}$ corresponding to Figure 9 does not have a well-defined Gauss map.

\section{BIBLIOGRAPHY}

[A] V. I. Arnold and A. N. Varchenko, Singularities of differentiable maps, Vol. 1, Birkhäuser, 1985.

[B] T. Banchoff, T. Gaffney, and C. McCrory, Cusps of Gauss maps, Pitman, 1982.

[GG] V. Golubitsky and V. Guillemin, Stable mappings and their singularities, Springer-Verlag, 1974.

[GS] V. Guillemin and S. Sternberg, Geometric asymptotics, Math. Surveys Monographs, Amer. Math. Soc., Providence, R.I., 1977.

[K1] M. Kossowski, Prescribing invariants for Lagrangian surfaces, Topology 31 (1992).

[K2] _ The asymptotic blow up of a surface in Euclidean 3-space, Geom. Dedicata 40 (1991).

[K3] __ Homotopy invariants for solutions to symplectic Monge Ampere equations, J. Differential Equations (to appear).

[M] D. Mond, On the classification of germs of maps from $\mathbf{R}^{2}$ to $\mathbf{R}^{3}$, Proc. London Math. Soc. (3) 50 (1985).

Department of Mathematics, University of South Carolina, Columbia, South CarOLINA 29208 